\title{
A MODIFIED POLARIMETRIC DECOMPOSTION FOR APPLICABILTY IN COMPLEX AGRICULTURAL ENVIRONMENT
}

\author{
Y.Dehghan-Soraki ${ }^{\mathrm{a} *}$, S.k. Saha ${ }^{\mathrm{b}}$, M. Kumari ${ }^{\mathrm{c}}$
}

\author{
${ }^{a}$ Remote Sensing Research Institute, Iranian Space Research Centre, Tehran, Iran - dehghan.you @gmail.com \\ ${ }^{\mathrm{b}}$ Indian Institute of Remote Sensing, Indian Space Research Organization, Dehradun, India - sksaha@iirs.gov.in \\ ${ }^{\mathrm{c}}$ Indian Institute of Remote Sensing, Indian Space Research Organization, Dehradun, India - mamta@iirs.gov.in
}

KEY WORDS: Polarimetric SAR, Decomposition, Land use/Land Cover, Agriculture, Crop inventory, Crop classification

\begin{abstract}
:
A new model based decomposition on coherency matrix is demonstrated for the analysis of polarimetric SAR data. In this method a first order Bragg scattering model was fitted to explain the Bragg scatter from a moderately rough surface; randomly oriented dipole for explaining the volume scattering from a canopy layer; and a rotated dihedral model for pair of orthogonal scatters in Copol and Crosspol channels. The distinctive nature of this model is: fitting a rotated dihedral model to the coherency matrix which provides an ability to distinguish the same double-bounce mechanism occurs in small size bunded cropland and in orthogonal manmade structures. The model is applied to Radarsat-2 full polarimetric data which was acquired in a complex agricultural landscape. The result from decomposition was quite effective in reducing the scattered double-bounce pollutions from bunded trees surrounding croplands with extra enhancement in decomposition of orthogonal as well as oblique manmade structures.
\end{abstract}

\section{INTRODUCTION}

Polarimetric synthetic aperture radar (POLSAR) can depict landmark imaging Characters better from ground - object polarized scattering mechanism and model. Therefore, it introduced new direction of the research of imaging recognition and classification. The most important observable measured by such radar systems is the $3 \times 3$ coherency matrix or equivalent 4 $\mathrm{x} 4$ Mueller matrix. Originated from work of Chandrasekhar on light scattering (Chandrasekhar, 1960), Huynen (Huynen, 1988) start to use practically target decomposition (TD) theorems for POLSAR image classification. Since then, there have been many other proposed decompositions for such applications(Cloude and Pottier, 1996).

The TD family can be broadly classified in two categories, namely: coherent (CDs) and incoherent decompositions (ICDs). Coherent decomposition attempts to decompose the coherent scattering matrix into the sum of elementary matrices (Cameron and Leung, 1990; Krogager, 1990). While these do not require estimates of the target coherency matrix, they are prone to problems from coherent speckle noise, from basis invariance and/or from lack of basis orthogonality. In fact, the scattering matrix cannot be employed to characterize, the polarimetric point of view, the so called distributed scatters; only second polarimetric observables like Hermitian average Coherency and Covariance matrices can be employed to analyse such scatters. Hence, the objective of ICDs would be to separate the so called Coherency or Covariance matrices as the combination of second order descriptors corresponding to simpler or canonical objects, presenting an easier and meaningful physical interpretation. Examples of such ICDs are Phenomenological Huynen Decomposition (Huynen, 1988), the Eigen value/Eigen vector based decomposition (Cloude and Pottier, 1996). An alternative to ICDs are Model based family of decomposition, which tries to use physical models of depolarization to determine the number and parameterization of each component. Respect to other form of decompositions, Model based decompositions are easy to fit on POLSAR datasets and they need less inputs to solve the forward scattering problem (Freeman and Durden, 1998; Yamaguchi et al., 2005).

However, the polarimetric decompositions and specially the Model based ones were successful to classify broad land use/land covers, but they still need to be enhanced for applicability in complex distributed landscape like agricultural lands. Assuming a wheat land in its booting stage, and a Cband Radar system, it is supposed to receive signal mainly in Crosspol channel. But, assuming the same target while surrounded by bund trees, the return from Wheat would be affected by strong double bounce return from tree trunk crop/Soil, in Copolarization channel (Figure 1). Generally, in conventional Model based decompositions, like Freeman or Yamaguchi decomposition, a dihedral model is fitted to explain this double bounce return, although the same model also apply for manmade or any vertical alignment to Radar line of sights (LOS). In this situation, the confusion between bunded small sized cropland and artificial manmade areas like villages cannot be inevitable.

Although the same response may derived from bunded crop lands and vertical manmade structures in current decomposition model, but by exploring the coherency matrix we noticed that: Crosspol signal which already known for volume scattering components, additionally contains rotated dihedral double bounce component, which rotates the received electromagnetic polarization. In order to explain this effect, a rotated dihedral model was defined to the coherency matrix and revised the current vegetation scattering model to account for additional even-bounce component in Crosspol channels.

* Corresponding author 


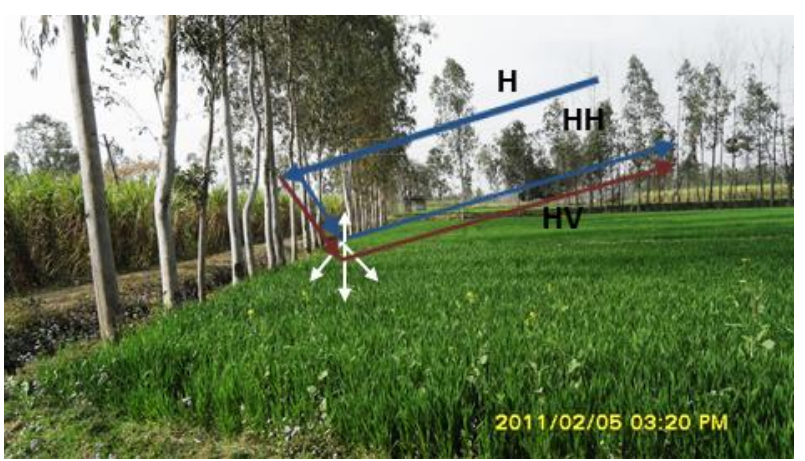

Figure 1 An image of typical cropland (Wheat crop) bunded by tall trees. Arrows on image shows the interaction of SAR polarized signals with tree trunk and Wheat crop in rotated Dihedral ordnances.

In order to explain this effect we define a rotated dihedral model to the coherency matrix and revised the current vegetation scattering model to account for additional even bounce component in Crosspol channels.

\section{MODEL DEVELOPMENT}

\subsection{Model Based Incoherent Polarimetric decomposition}

An alternative approach to incoherent decomposition is Model based polarimetric decomposition. In fact model based family of decompositions uses physical models of depolarization to determine the number and parameterization of each component (Freeman and Durden, 1998; Sato et al., 2012; van Zyl, 1989; Yamaguchi et al., 2005). The general starting point for this approach is to identify the main components to any backscattering scenarios as: surface, double bounce and volume scatters. The simplest form involves a generic two-layer approach, with a volume layer of particles (vegetation, snow, and so on) above a non-penetrable boundary or surface. The total average backscatter is then determined from a $3 \times 3$ coherency matrix composed of three main elements, as shown in equation (1):

$$
[T]=\left[T_{S}\right]+\left[T_{D}\right]+\left[T_{V}\right]
$$

The first component $-\mathrm{S}$ consist of Bragg surface scatter (Freeman and Durden, 1998) modelling form slightly rough surface in which the cross polarized component is negligible. The coherency matrix obtained from respected scattering matrix is given by:

$$
\begin{aligned}
& \left\langle\left[T_{S}\right]\right\rangle=\left[\begin{array}{ccc}
1 & a^{*} & 0 \\
a & |a|^{2} & 0 \\
0 & 0 & 0
\end{array}\right] \\
& \text { Where } \quad a=\frac{R_{H}-R_{V}}{R_{H}+R_{V}},|a|<1
\end{aligned}
$$

In equation (2) $R_{H}$ and $R_{V}$ are the Fresnel reflection coefficient for horizontally and vertically polarized wave and $<>$ represent the ensemble average over the whole data. The second component-D in equation (1) - involves a multiple scattering interaction between the surface reflection and volume-scattering elements that return the reflected signal back to the observer. For double bounce scattering mechanism (Freeman and Durden, 1998) :

$$
\begin{aligned}
& \left\langle\left[T_{D}\right]\right\rangle=\left[\begin{array}{ccc}
|b|^{2} & b & 0 \\
b^{*} & 1 & 0 \\
0 & 0 & 0
\end{array}\right] \\
& b=\frac{e^{i 2 \gamma_{H}} R_{G H} R_{T H}+e^{i 2 \gamma_{V}} R_{G V} R_{T V}}{e^{i 2 \gamma_{H}} R_{G H} R_{T H}-e^{i 2 \gamma_{V}} R_{G V} R_{T V}},|b|<1
\end{aligned}
$$

Where, $\mathrm{R}_{\mathrm{GH}}, \mathrm{R}_{\mathrm{TH}}$ and $\mathrm{R}_{\mathrm{GV}}, \mathrm{R}_{\mathrm{TV}}$ represent the reflection coefficients of the horizontal and vertical for horizontal and vertical polarization, from ground and trees respectively. The $\gamma_{\mathrm{H}}$ and $\gamma_{\mathrm{v}}$ are propagation attenuation factor.

The third component $-\mathrm{V}$ in equation (1) - is direct volume scattering from the top layer (vegetation layer) itself. This scattering is modelled by considering the vegetation canopy as a cloud of randomly oriented thin cylinder like scatters (Yamaguchi et al., 2006). The coherency matrix can be represented as:

$$
\left[T_{V}\right]=\frac{1}{4}\left[\begin{array}{lll}
2 & 0 & 0 \\
0 & 1 & 0 \\
0 & 0 & 1
\end{array}\right]
$$

\subsection{Proposed modified Polarimetric decomposition}

Respect to dominant scattering mechanism which can happen in an agricultural environment, we can re-write the coherency matrix based on Pauli matrices as:

$$
\langle[T]\rangle=\left[\begin{array}{lll}
T_{11} & T_{12} & T_{13} \\
T_{21} & T_{22} & T_{23} \\
T_{31} & T_{32} & T_{33}
\end{array}\right]
$$

$$
\begin{aligned}
T_{11} & =\frac{1}{2}\left\langle\left|S_{H H}+S_{V V}\right|^{2}\right\rangle \\
T_{12} & =\frac{1}{2}\left\langle\left(S_{H H}+S_{V V}\right)\left(S_{H H}-S_{V V}\right)^{*}\right\rangle \\
T_{13} & =\left\langle\left(S_{H H}+S_{V V}\right) S_{H V}^{*}\right\rangle \\
T_{21} & =\frac{1}{2}\left\langle\left(S_{H H}-S_{V V}\right)\left(S_{H H}+S_{V V}\right)^{*}\right\rangle \\
T_{22} & =\frac{1}{2}\left\langle\left|S_{H H}-S_{V V}\right|^{2}\right\rangle \\
T_{23} & =\left\langle\left(S_{H H}-S_{V V}\right) S_{H V}^{*}\right\rangle
\end{aligned}
$$




$$
\begin{aligned}
& T_{31}=\left\langle S_{H V}\left(S_{H H}+S_{V V}\right)^{*}\right\rangle \\
& T_{32}=\left\langle S_{H V}\left(S_{H H}-S_{V V}\right)^{*}\right\rangle \\
& T_{33}=\left\langle 2\left|S_{H V}\right|^{2}\right\rangle
\end{aligned}
$$

From section 2.1, for single bounce or surface scattering model, the coherency matrix is:

$$
\left\langle\left[T_{S}\right]\right\rangle=\left[\begin{array}{lll}
1 & 0 & 0 \\
0 & 0 & 0 \\
0 & 0 & 0
\end{array}\right]
$$

For double bounce scattering model we fitted rotated dihedral plane with an orientation angle $\beta$, since this is more suits with backscatter from surface/crops-tree trunk than a simple dihedral model:

$$
\left\langle\left[T_{D}\right]\right\rangle=\left[\begin{array}{ccc}
0 & 0 & 0 \\
0 & \cos ^{2} 2 \beta & \frac{-\sin 4 \beta}{2} \\
0 & \frac{-\sin 4 \beta}{2} & \sin ^{2} 2 \beta
\end{array}\right]
$$

And finally for volume scattering model and regards to randomly oriented dipole the coherency matrix can be represented as (Yamaguchi et al., 2006):

$$
\left[T_{V}\right]=\frac{1}{4}\left[\begin{array}{lll}
2 & 0 & 0 \\
0 & 1 & 0 \\
0 & 0 & 1
\end{array}\right]
$$

Now from equations (1), (6), (7) and (8):

$$
\langle[T]\rangle=P_{s}\left[\begin{array}{ccc}
1 & 0 & 0 \\
0 & 0 & 0 \\
0 & 0 & 0
\end{array}\right]+P_{d}\left[\begin{array}{ccc}
0 & 0 & 0 \\
0 & \cos ^{2} 2 \beta & \frac{-\sin 4 \beta}{2} \\
0 & \frac{-\sin 4 \beta}{2} & \sin ^{2} 2 \beta
\end{array}\right]
$$$$
+\frac{P_{v}}{4}\left[\begin{array}{lll}
2 & 0 & 0 \\
0 & 1 & 0 \\
0 & 0 & 1
\end{array}\right]
$$

$$
\begin{aligned}
& P_{S}+2 P_{V}=\frac{1}{2}\left\langle\left|S_{H H}+S_{V V}\right|^{2}\right\rangle \\
& P_{d} \cos ^{2} 2 \beta+P_{v}=\frac{1}{2}\left\langle\left|S_{H H}-S_{V V}\right|^{2}\right\rangle \\
& P_{d} \sin ^{2} 2 \beta+P_{v}=\left\langle 2\left|S_{H V}\right|^{2}\right\rangle
\end{aligned}
$$

Four unknown parameters in equation (10) i.e. $\mathrm{P}_{\mathrm{s}}, \mathrm{P}_{\mathrm{d}}, \mathrm{P}_{\mathrm{v}}$ and the orientation angle $\beta$ along with four equations can be derived mathematically. Now, we can divide the power of double bounce component $\left(\mathrm{P}_{\mathrm{d}}\right)$ into two powers i.e. $\mathrm{P}_{\mathrm{d}} \cos ^{2} 2 \beta$ from Copol $\left(\mathrm{P}_{\mathrm{d}}\right)$ and $\mathrm{P}_{\mathrm{d}} \sin ^{2} 2 \beta$ from Crosspol $\left(\mathrm{P}_{\mathrm{dv}}\right)$ components of scattering matrix. These two above mentioned components are the key elements which provide us new capabilities to distinguish the different kind of double bounce scatters respect to their effects on change of polarization. We outlined the new decomposition method as a modification to the decomposition developed by Freeman and Durden (Freeman and Durden, 1998) as well as method developed by Yamaguchi (Yamaguchi et al., 2010).

\section{EXPERIMENT RESULTS AND ANALYSIS}

The new developed poalimetric decomposition were applied on polarimetric dataset, taken over part of Saharanpur city in northern India and in $5^{\text {th }} \mathrm{Feb}, 2011$. The quad-pol data set used is C-band Radarsat-2 (RS-2) data in Wide-fine mode. In order to reduce the calculation cost and preserve the complexity of the targets, in this work we selected a small area on RS-2 image as a region of interest (ROI) for processing and implementation. For the sake of comparison between current decomposition and our modified decomposition method, firstly we applied the decomposition proposed by Yamaguchi (Yamaguchi et al., 2006) on above mentioned dataset (Figure 2).

The complexity of region and effects of speckle are much clear in the resulted RGB image for Yamaguchi decomposition (Figure 2). Contribution of different kind of scatters also calculated and is shown in Figure 3 for selected patches, indicated on Figure 2. Accordingly, Figure 3 showed the major contribution of double bounce scatters in selected crop land (patch B), which is the best representation of typical cropland in this area. The Reason for these scattering phenomena is related to the embanking tall trees which surrounded the crop lands. This can make confusion in any polarimetric classification algorithm to effectively classify the information classes.

According to the results which we obtained in our previous work (Dehghan-Soraki et al., 2011), even with the help of speckle filtering also, we are not able to illuminate the double bounced pixels surrounded by volume clusters and succeed to reliable classification results. The confusion is more critical compare with the same double-bounce response which already exists in orthogonal manmade structures to radar line of sight, like rural buildings. In fact, in conventional decompositions, small sized bunded croplands as well as orthogonal manmade structures are decomposing to same double-bounce component.

From equation (5) and (9):

$$
\frac{-\sin 4 \beta}{2}=\left\langle\left(S_{H H}-S_{V V}\right) S_{H V}{ }^{*}\right\rangle
$$




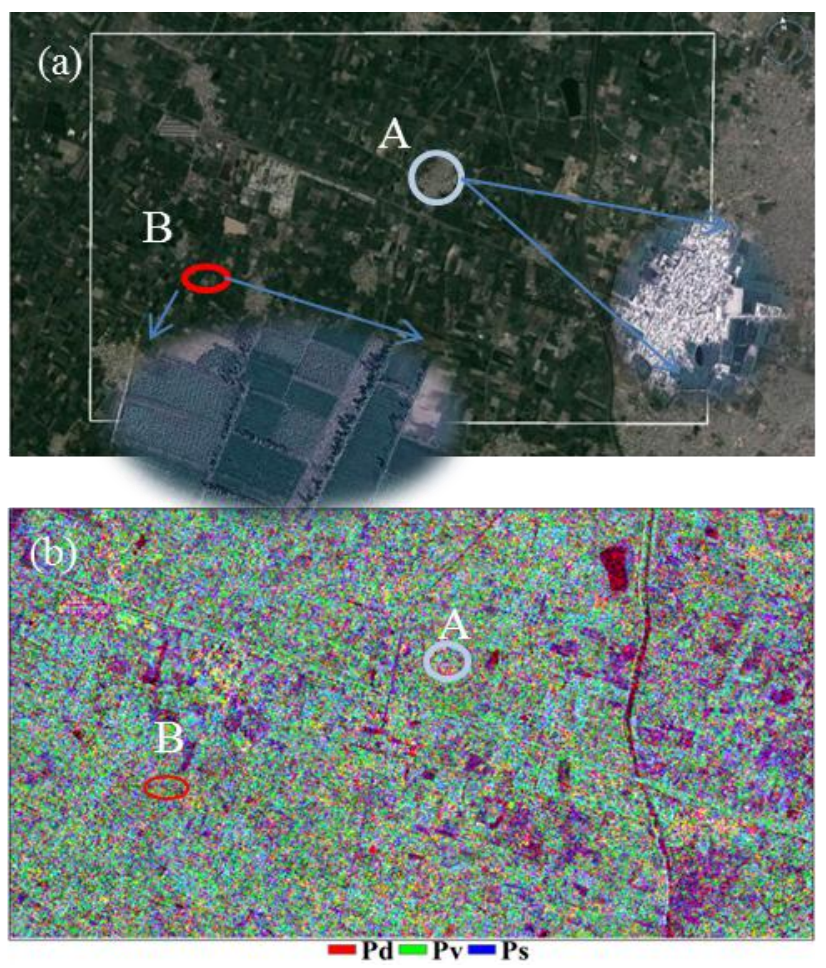

Figure 2 Google Earth image of the study area (a), Yamaguchi decomposition of RS-2 Polsar image of respected area, Circle A and B are selected patches for manmade and crop lands respectively (b).

Oblique manmade structures to Radar LOS are another problem that made miss-decomposition on Polsar data. The orthogonal manmade structures are mainly decomposing to double-bounce classes while in oblique cases, they known to be decomposed to volume classes (Yamaguchi et al., 2010). As it is clear in Figure 2 also, in proportion to Patch-A, rural buildings were mainly decomposed to volume classes by $56 \%$ against $37 \%$ of doublebounce ones.

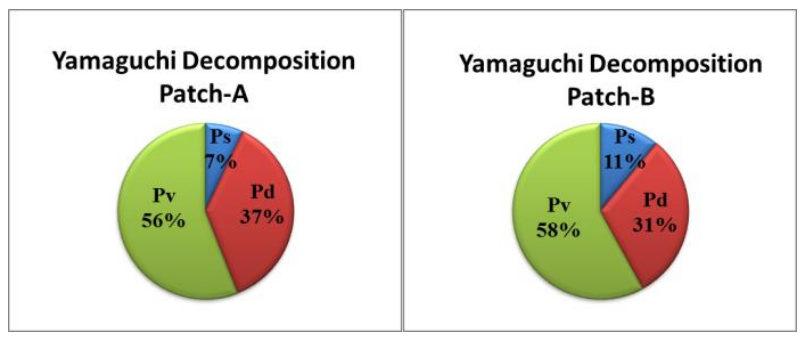

Figure 3 Percentages of different kind of scattering powers for selected patches in Figure 2.

After exploring some defects of previous decomposed images, the new developed decomposition applied on same Polsar dataset (Figure 4). Extracting and examining the spatial distribution of powers for two different double bounce classes i.e., $\mathrm{P}_{\mathrm{d}}$ from Copol and $\mathrm{P}_{\mathrm{d}}$ from Crosspol $\left(\mathrm{P}_{\mathrm{dv}}\right)$, provides an ability to understand the major kind of scatters which are responsible for these different kinds of responses.

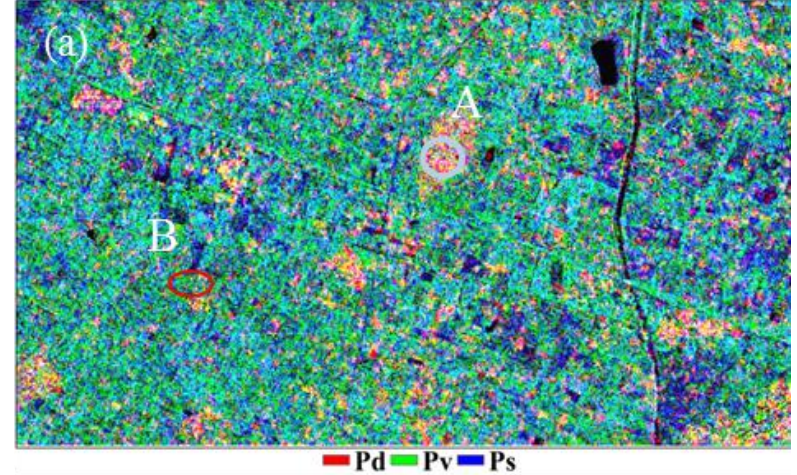

(b)

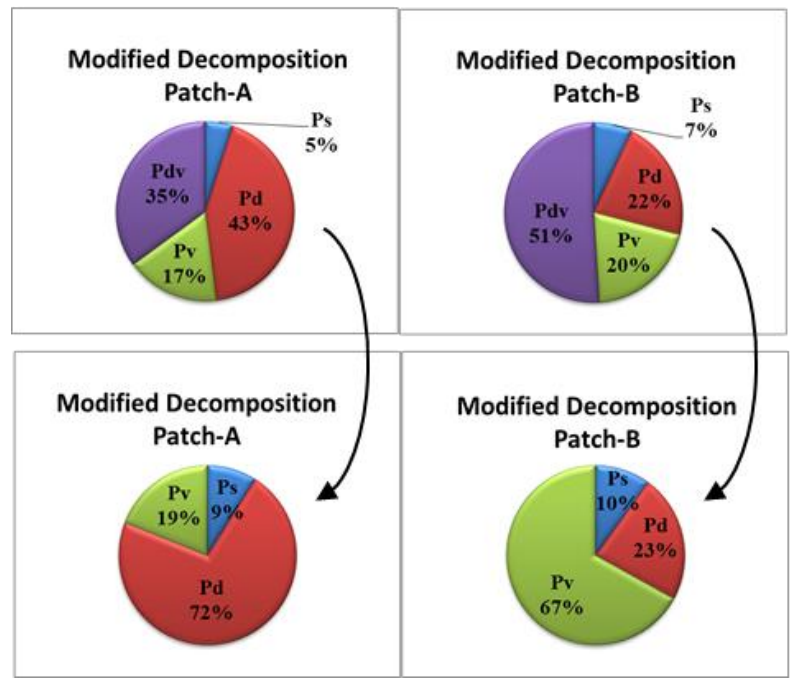

Figure 4 RGB image from new Modified polarimetric decomposition (a), corresponding percentage of different kind of scattering powers and changes after reassignment of power for Patch A and B (b).

According to Figure 4-b $\mathrm{P}_{\mathrm{d}}$ is both common in manmade structures and agricultural lands, while $\mathrm{P}_{\mathrm{dv}}$ seems to be more frequent in agricultural areas. Subsequently, based on findings we added an extra decision rule to the decomposition which proposed by Yamaguchi, et al (Yamaguchi et al., 2010); where we added the power of $P_{d v}$ to $P_{v}$ or $P_{s}$ wherever the frequency or the mean power of $P_{d v}$ was greater than $P_{d}$ in specified kernel (here is 5by5), and it added to $P_{d}$ wherever this inequality was less. The RGB image in Figure 4-a, itself is the best indicators for enhancement which obtained both in croplands as well as in settlement areas (here villages).

Although overall enhancement on different land covers achieved, but still there are some red spots related to the double bounce like scatters over the crop lands. By exploring those regions it already proved that; thos $3 \mathrm{e}$ spots are related to individual desperate artificial structures whether in the form of farmer's building or in any form of vertical alignments. These kinds of targets are known to have strong Copol responses in Polsar backscattering powers, so after applying the new decomposition also they still remain in double-bounce classes. In fact, solving these issues may still constrained to have a Polsar images with more enhanced spatial resolution. 


\section{CONCLUDING REMARKS}

A modified technique for decomposition of Polsar image acquired in a complex agricultural landscape has been presented. Fitting a rotated dihedral model to the coherency matrix has provided unique ability to distinguish various kinds of even-bounce returns on received power. In addition to the double-bounce $\left(\mathrm{P}_{\mathrm{d}}\right)$ sacatterers in Copolarized channel, another even-bounce component was extracted from Crosspolarized channel $\left(\mathrm{P}_{\mathrm{dv}}\right)$. By spatially exploring the decomposed products, extinctive behaviour of $\mathrm{P}_{\mathrm{dv}}$ and $\mathrm{P}_{\mathrm{d}}$ were observed respected to different land covers. It has seen that, in comparison with $P_{d}$, the frequency and mean power of $\mathrm{P}_{\mathrm{dv}}$ is more common in croplands respect manmade structured areas. Accordingly, a modification was introduced to decomposition which proposed by Yamaguchi (Yamaguchi et al., 2005), in reassignment and re-allocation of $\mathrm{P}_{\mathrm{dv}}$ to the surface power (Ps), volume power $\left(\mathrm{P}_{\mathrm{v}}\right)$ and finally to general double bounce power $\left(\mathrm{P}_{\mathrm{d}}\right)$ from Copolarized channels.

In combination with Yamaguchi decomposition, the modified decomposition is quite simple and effective. The method was successfully carried on Radarsat-2 quad-pol dataset and effectively discriminated the small bunded croplands from manmade structures. In combination with de-orientation method of Yamaguchi (Yamaguchi et al., 2010) also, oblique manmade structures were successfully highlighted and decomposed to double-bounce component. In future work, we intend to use the resulted decomposed images as classification features along with other polarimetric observables to allow differentiation between different kinds of crops. We also intend to extend and automatize the scope of the model to various kinds of SAR systems with different frequencies (X, L and $\mathrm{P}$ band) as well as multiple incidence angles.

\section{ACKNOWLEDGEMENT}

This work has been carried out under the grant by Centre for Space Science and Technology Education in Asia and The Pacific (CSSTEAP), Affiliated by United Nations. The authors would also like to thanks Indian Institute of Remote Sensing (IIRS) and ISRO for its scientific as well as field supports.

\section{REFRENCES}

Cameron, W.L., Leung, L.K., 1990. Feature motivated polarization scattering matrix decomposition, Proc. IEEE Int. Radar Con, Arlington, VA, p. 9.

Chandrasekhar, S., 1960. Radiative Transfer. New York: Dover, New York: Dover.

Cloude, S.R., Pottier, E., 1996. A Review of Target Decomposition Theorems in Radar Polarimetry. IEEE TRANSACTIONS ON GEOSCIENCE AND REMOTE SENSING 34, p. 21.

Dehghan-Soraki, Y., Saha, S.K., Kumari, M., 2011. Use of Satellite SAR Polarimetry in crop Inventory- A case study., Centre for Space Science and Technolgy Education in Asia and The Pacifics, Dehradun-India.

Freeman, A., Durden, S., 1998. A Three-Component Scattering Model for Polarimetric SAR Data. IEEE TRANSACTIONS ON GEOSCIENCE AND REMOTE SENSING 36, pp. 963-973.
Huynen, J.R., 1988. Comments on radar target decomposition theorems, P. Q. Res. CA, Rep, Los Altos Hills.

Krogager, E., 1990. A new decomposition of the radar target scattering matrix. Electron. Lett 26, p. 2.

Sato, A., Yamaguchi, Y., Singh, G., Park, S.-E., 2012. FourComponent Scattering Power Decomposition With Extended Volume Scattering Model. IEEE Geoscience and Remote Sensing Letters 9, p. 5.

van Zyl, J.J., 1989. Unsupervised classification of scattering behaviour using radar polarimetry data. IEEE Transactions on Geoscience and Remote Sensing 27, p. 9.

Yamaguchi, Y., Moriyama, T., Ishido, M., Yamada, H., 2005. Fourcomponent scattering model for polarimetric SAR image decomposition. IEEE Transactions on Geoscience and Remote Sensing 43, p. 8.

Yamaguchi, Y., Sato, A., Sato, R., Yamada, H., M. Boerner, W., 2010. FOUR-COMPONENT SCATTERING POWER DECOMPOSITION WITH ROTATION OF COHERENCY MATRIX. IEEE GEOSCIENCE AND REMOTE SENSING 49, pp. 2251-2258.

Yamaguchi, Y., Yajima, Y., Yamada, H., 2006. A FourComponent Decomposition of POLSAR Images Based on the Coherency Matrix. IEEE GEOSCIENCE AND REMOTE SENSING LETTERS 3, pp. 292-296. 